Theatre Recherches

Research théâtrales

in Canada au Canada
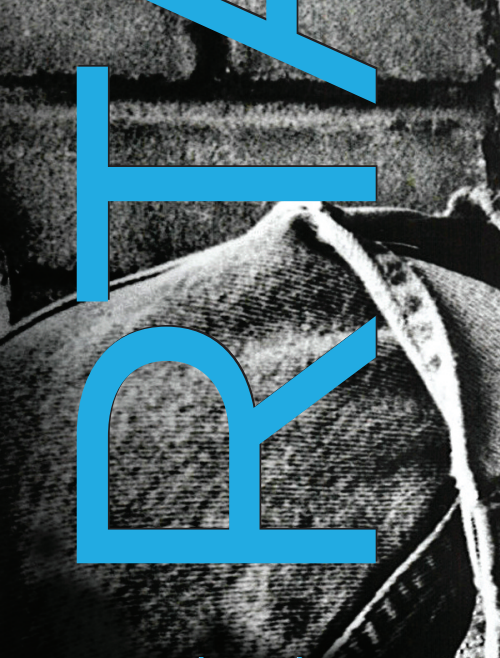

VOL 35 / No 3 / 2014

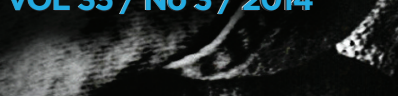

$\operatorname{lin}^{2}$
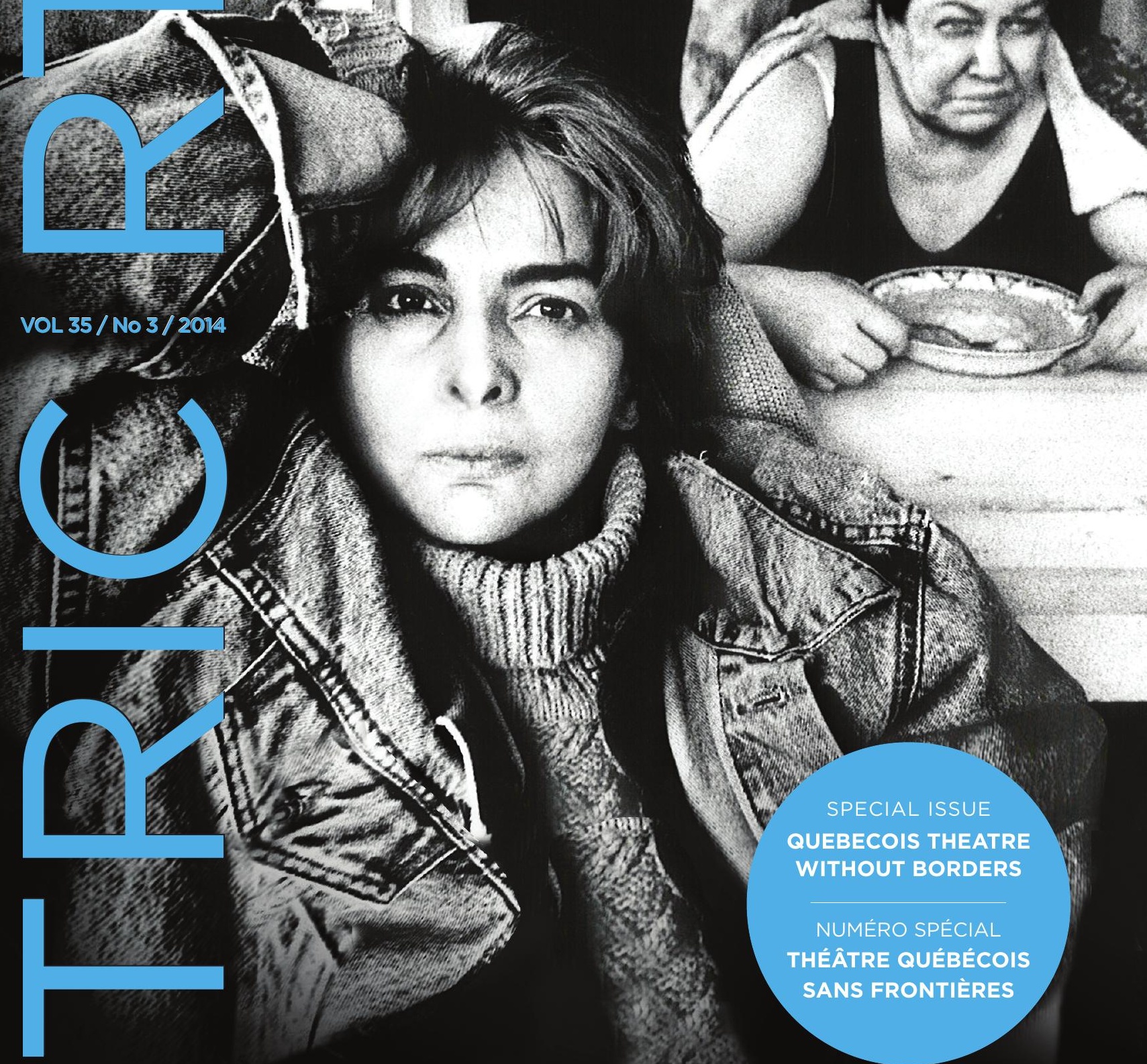

ats
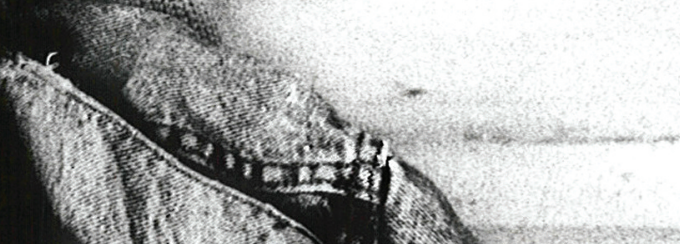

(2)

at 1 a

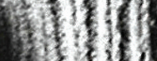

I)

soln

1.9.

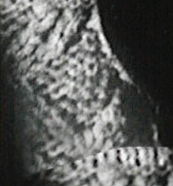

2710

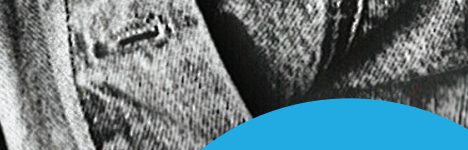
1.4?

(n)

1.1

'HW SPECIAL ISSUE QUEBECOIS THEATRE WITHOUT BORDERS

NUMÉRO SPÉCIAL

THÉÂTRE QUÉBÉCOIS SANS FRONTIËRES 


\section{DIRECTEURS DE LA REVUE}

EDITOR / RÉDACTEUR VOL 35 / No 3

Marlis Schweitzer, York University

\section{ASSOCIATE FRENCH EDITOR / RÉDACTRICE ADJOINTE FRANCOPHONE}

Louise Ladouceur, Campus St-Jean, Université de I'Alberta

BOOK REVIEW EDITOR / RÉDACTRICE COMPTES RENDUS

Michelle MacArthur, University of Toronto

\section{EDITORIAL MANAGEMENT / DIRECTION DE RÉDACTION}

Barry Freeman, Executive Editor, University of Toronto

Shelley Liebembuk, Senior Editorial Assistant, University of Toronto

Kelsy Vivash, Senior Editorial Assistant, University of Toronto

Sonya Malaborza, Translator / traductrice

Milane Pridmore-Franz, Translator / traductrice

\section{EDITORIAL BOARD / COMITÉ DE RÉDACTION}

Roberta Barker, Dalhousie University

George Belliveau, University of British Columbia

Kym Bird, York University

Susan Bennett, University of Calgary

Louise Forsyth, University of Saskatchewan

Helen Gilbert, Royal Holloway, University of London

Reid Gilbert, University of British Columbia; Capilano U (Emeritus)

Erin Hurley, McGill University

Ric Knowles, University of Guelph

André Loiselle, Carleton University

Glen Nichols, Mount Allison University

Roger Parent, Campus St-Jean, University of Alberta

Gregory J. Reid, Université de Sherbrooke

Jonathan Rittenhouse, Bishop's University

Denis Salter, McGill University

Kim Solga, University of Western Ontario

\section{MANAGEMENT BOARD / COMITÉ DIRECTEUR}

Jenn Stephenson, Chair / Président, Queen's University

Annie Gibson, Playwrights Canada Press

Kim Solga, University of Western Ontario

Stephen Johnson, CATR / ACRT, University of Toronto

Robin C. Whittaker, Online Coordinator, Saint Thomas University

\section{FOUNDING EDITORS / RÉDACTEURS FONDATEURS}

Ann Saddlemyer, University of Toronto

Richard Plant, University of Toronto

JOURNAL DESIGN

Louis Duarte 


\section{Contents / Sommaire}

\section{INTRODUCTION / PRÉSENTATION}

295 Hervé Guay and Erin Hurley Quebecois Theatre without Borders / Théâtre québécois sans frontières

\section{ARTICLES}

\section{Sylvain Schryburt}

Esquisse d'une sociologie des réseaux festivaliers. Le cas du Festival TransAmériques de Montréal

317 Andrea Pelegrí Kristić Daniel Danis en traduction : problèmes de traduction du Chant du Dire-Dire en espagnol chilien

330 Melissa Poll

Adapting "Le Grand Will" in Wendake: Ex Machina and the Huron-Wendat Nation's La Tempête

352 Karen Fricker

"The Simple Question of Ireland": La Reine de beauté de Leenane in Montreal

\section{FORUM: BEYOND BORDERS}

378 Dragana Varagic and Kathleen Irwin Words... Words... Words: The Novel, the Play, the Production
387 Samer Al-Saber

Arabic Facts in Palestine: Clashing Hybridities in Transnational Cultural Production

\section{Patrick Blenkarn}

D through Z: Politics in the Making

\section{BOOK REVIEWS/ COMPTES RENDUS}

403 Brad Krumholz

R. Darren Gobert, The Mind-Body Stage: Passion and Interaction in the Cartesian Theater

405 Louise Ladouceur

Sylvain Schryburt, De l'acteur vedette au théâtre de festival. Histoire des pratiques scéniques montréalaises (1940-1980)

407 Yana Meerzon

Michelle MacArthur, Lydia Wilkinson, Keren Zaiontz, eds., Performing Adaptations. Essays \& Conversations on the Theory and Practice of Adaptation

409 Katherine Zien

Natalie Alvarez, ed., Fronteras Vivientes: Eight Latina/o Canadian Plays

Latinalo Canadian Theatre and Performance 


\section{CONTRIBUTORS/} COLLABORATEURS

418 Guidelines for Submissions/ Avis aux correspondants

420 Submission Response Schedule/ Calendrier des interventions

Cover / Couverture:

Micheline Bernard (left) and Denise Gagnon (right) as Maureen and Mag in La Reine de beauté de Leenane by Martin McDonagh, translated by Fanny Britt and directed by Martin Faucher, at Théâtre La Licorne. Photo by Rolline Laporte. 